\title{
Propuesta de un PRoceso de investigación Cuantitativa. APLICACIÓN EN LA CARACTERIZACIÓN DE LAS MYPES PRODUCTORAS
}

\section{DE SOFTWARE}

\author{
Miriam Elizabeth Amable Ciudad \\ Escuela de Ingeniería de la Universidad de Lima. Lima, Perú
}

Recibido: 13 de octubre de 2015 / Aprobado: 30 de octubre de 2015

\begin{abstract}
Resumen
En este artículo se resalta tanto el proceso de investigación cuantitativa como su aplicación para lograr el objetivo de identificar el nivel de calidad en las mypes productoras de software. El presente trabajo está dirigido a los estudiantes en su rol como investigadores, teniendo en cuenta que uno de los objetivos estratégicos de las instituciones de educación superior es promover la cultura de la investigación. Es de esa manera que se logrará una significativa diferencia y se adquirirá una mejor formación, lo cual les permitirá ser más competitivos. El proceso de investigación cuantitativa facilita la realización de métodos primarios que hacen posible obtener la caracterización de la mypes productoras de software de Lima, y así establecer el nivel de calidad de este tanto en sus procesos como en sus productos.
\end{abstract}

Palabras clave: proceso de investigación cuantitativa / calidad de software / encuesta / mypes / producto software/ procesos de software

\section{Proposal for a Quantitative Research Process. Applying the Characteristics of the Software Producing Mypes (Micro and Small Enterprises)}

\section{Summary}

This article highlights both the quantitative research process and its implementation to achieve the objective of identifying the level of quality in software producing mypes. The current project is aimed at students in their role as researchers, considering that one of the strategic objectives of the institutions of higher education is to promote the culture of research, look for differences in findings, obtain superior training and to be more competitive. The quantitative research process gives way to the realization of the primary methods in obtaining the characterization of software producing mypes in Lima. This allows one to identify the quality of their software.

Key words: quantitative research process / software quality / survey / mypes / software product I software processes 


\section{Introducción}

La educación superior, según T. C. A. Bernal (2010), "debe estar inmersa en la producción de conocimiento que, desde un enfoque integral e interdisciplinario, permita analizar y resolver los problemas actuales de la sociedad así como proveer estrategias que dinamicen los avances tecnológicos" (p. 9).

En la Universidad de Lima, esta labor de investigación es promovida tanto por el Instituto de Investigación Científica (IDIC) como en las asignaturas Seminario de Tesis I y Seminario de Tesis II, cuyo propósito en conjunto es desarrollar trabajos de investigación. Por ello es importante, en cualquier contexto, seguir el proceso formal de metodología de la investigación, para lograr un resultado deseado, confiable y de calidad. Según Bernal (2010), en el proceso de investigación científica:

El método cuantitativo se fundamenta en la medición de las características de los fenómenos sociales, lo cual supone deriva de un marco conceptual pertinente al problema analizado, una serie de postulados que expresen relaciones entre las variables estudiadas de forma deductiva. Este método tiende a generalizar y normalizar resultados. (p. 60)

En el enfoque cuantitativo se someten a prueba en la realidad cuando se aplica un diseño de investigación, se recolectan datos con uno o varios instrumentos de medición, y se analizan e interpretan esos mismos datos (Hernández, Fernández y Baptista, 2010).

Además de plantear un problema de manera adecuada, sustentado en la teoría y los resultados empíricos previos, se requiere también la utilización adecuada de técnicas de recolección de datos y de análisis estadísticos pertinentes, lo mismo que el análisis y la correcta interpretación de los resultados con base en los conocimientos que sirvieron de sustento a la investigación (Hernández et al., 2010). Por ello, en la investigación realizada en el IDIC (Amable, Millones y Checa (2014) se siguió el proceso de investigación cuantitativa para obtener la caracterización de las mypes productoras de software y determinar sus fortalezas y debilidades, y en este último punto identificar sus problemas y proponer mejoras a dichas organizaciones.

En este artículo se presenta el proceso de investigación cuantitativa desde la perspectiva de varios autores, eligiéndose un proceso con sus diversas etapas y asociado a ellas lo que se desarrolló en la investigación del IDIC; esto de manera ilustrativa, para que sirva de guía para otros trabajos de investigación.

1.

\section{Proceso de la investigación cuantitativa}

En Hernández et al. (2010) se define la investigación como "un conjunto de procesos sistemáticos, críticos y empíricos que se aplican al estudio de un fenómeno" (p. 4). 
Existen dos enfoques claramente definidos: el cuantitativo y el cualitativo, los cuales "son procesos cuidadosos, metódicos y empíricos (...) para generar conocimiento" (p. 4). El enfoque cuantitativo es un proceso secuencial y probatorio.

Asimismo, señala que "cada etapa precede a la siguiente y no se puede 'brincar o eludir' pasos, el orden es riguroso, aunque, desde luego, podemos redefinir alguna fase" (Hernández et al., 2010, p. 4). Teniendo en cuenta esta definición se revisaron tres textos adicionales al de los autores citados, en el que se considera la redefinición de alguna fase: el primero es el de Alarcón (2008), el cual presenta el proceso de investigación del comportamiento bajo la perspectiva psicológica; los otros dos textos son Bernal (2010) y Valderrama (2014). En la tabla 1 se muestra una comparación de las etapas propuestas por los autores referidos, para el proceso de investigación cuantitativa.

En Valderrama (2014) se destaca una herramienta llamada 'matriz de consistencia' que el investigador elabora para confirmar la lógica tanto vertical como horizontal de los elementos que conforman el trabajo de investigación, al igual que la trazabilidad desde la definición del problema, objetivos, hipótesis, variables y diseño metodológico; elementos definidos desde el inicio hasta el final.

2.

\section{Propuesta de un proceso de investigación}

Teniendo en cuenta las fuentes referidas en la sección 2 de este artículo, se considera una propuesta de un proceso de investigación cuantitativa basado en nueve etapas:

Etapa 1: selección del tema de investigación o idea de la investigación

Etapa 2: definición del problema de la investigación

Etapa 3: establecimiento de los objetivos generales y específicos

Etapa 4: establecer la justificación y delimitación de la investigación

Etapa 5: definición del tipo de investigación

Etapa 6: establecimiento del marco de referencia de la investigación

Etapa 7: proceso de realización de la encuesta

Etapa 8: recolección de los datos cuantitativos

Etapa 9: analizar los resultados

A continuación se explican cada una de las etapas mencionadas:

\subsection{Selección del tema de investigación o idea de la investigación}

Tanto en Bernal (2010) como en Hernández et al. (2010) se indica que esta primera etapa nace de la observación del mundo real, identificando -como se refiere en Hernández et al. (2010) - un "primer acercamiento a la realidad" (p. 26), percibiendo 
Tabla 1

Comparación de las etapas propuestas para el proceso de investigación cuantitativa

\begin{tabular}{|c|c|c|c|}
\hline Hernández et al. (2010) & Alarcón (2008) & Bernal (2010) & Valderrama (2014) \\
\hline I. Idea & I. Tema de investigación & I. Tema de investigación & I. Título de investigación \\
\hline $\begin{array}{l}\text { II. Planteamiento } \\
\text { del problema }\end{array}$ & $\begin{array}{l}\text { II. Formulación } \\
\text { del problema }\end{array}$ & $\begin{array}{l}\text { II. Problema de } \\
\text { la investigación }\end{array}$ & $\begin{array}{l}\text { II. El problema } \\
\text { de la investigación }\end{array}$ \\
\hline $\begin{array}{l}\text { a. Establecer los objetivos } \\
\text { de investigación }\end{array}$ & $\begin{array}{l}\text { III. Formulación } \\
\text { de una hipótesis }\end{array}$ & & $\begin{array}{l}\text { a. Planteamiento } \\
\text { del problema }\end{array}$ \\
\hline $\begin{array}{l}\text { b. Desarrollar las preguntas } \\
\text { de investigación }\end{array}$ & & $\begin{array}{l}\text { III. Objetivos } \\
\text { de la investigación }\end{array}$ & $\begin{array}{l}\text { b. Formulación del } \\
\text { problema general y de } \\
\text { problemas específicos }\end{array}$ \\
\hline $\begin{array}{l}\text { c. Justificar la investigación } \\
\text { y analizar su viabilidad }\end{array}$ & & $\begin{array}{l}\text { IV. Justificación y delimi- } \\
\text { tación de la investigación }\end{array}$ & $\begin{array}{l}\text { c. Justificación } \\
\text { de la investigación }\end{array}$ \\
\hline $\begin{array}{l}\text { d. Evaluar las deficiencias en el } \\
\text { conocimiento del problema }\end{array}$ & & V. Tipos de investigación & $\begin{array}{l}\text { d. Alcances } \\
\text { de la investigación }\end{array}$ \\
\hline \multirow{4}{*}{$\begin{array}{l}\text { III. Desarrollo de la perspec- } \\
\text { tiva teórica: revisión de la } \\
\text { literatura y construcción del } \\
\text { marco teórico }\end{array}$} & $\begin{array}{l}\text { IV. Revisión de la } \\
\text { literatura sobre } \\
\text { el problema }\end{array}$ & $\begin{array}{l}\text { VI. Marco de referencia } \\
\text { de la investigación }\end{array}$ & $\begin{array}{l}\text { III. Marco } \\
\text { de referencia }\end{array}$ \\
\hline & & & a. Antecedentes \\
\hline & & & b. Marco teórico \\
\hline & & & c. Marco conceptual \\
\hline $\begin{array}{l}\text { IV. Definir el alcance } \\
\text { de la investigación }\end{array}$ & $\begin{array}{l}\text { V. Selección del método } \\
\text { de investigación }\end{array}$ & $\begin{array}{l}\text { VII. Hipótesis } \\
\text { de la investigación }\end{array}$ & IV. Hipótesis \\
\hline V. Formulación de hipótesis & a. Selección del diseño & $\begin{array}{l}\text { VIII. Diseño experimental } \\
\text { de la investigación }\end{array}$ & a. Hipótesis general \\
\hline $\begin{array}{l}\text { VI. Desarrollo del diseño de } \\
\text { investigación }\end{array}$ & b. Selección de la muestra & $\begin{array}{l}\text { IX. Determinación de la } \\
\text { población y la muestra } \\
\text { objeto de estudio }\end{array}$ & b. Hipótesis específicas \\
\hline $\begin{array}{l}\text { VII. Definición y selección de } \\
\text { la muestra }\end{array}$ & $\begin{array}{l}\text { c. Preparación de los } \\
\text { instrumentos para } \\
\text { recoger los datos }\end{array}$ & $\begin{array}{l}\text { X. Obtención de la } \\
\text { información. Recopilación }\end{array}$ & c. Hipótesis nula \\
\hline $\begin{array}{l}\text { VIII. Recolección de los datos } \\
\text { cuantitativos }\end{array}$ & $\begin{array}{l}\text { VI. Conducción de los } \\
\text { exámenes: obtención } \\
\text { de los datos }\end{array}$ & $\begin{array}{l}\text { XI. Procesamiento de la } \\
\text { información. Datos }\end{array}$ & $\begin{array}{l}\text { d. Variables. Definición } \\
\text { conceptual }\end{array}$ \\
\hline $\begin{array}{l}\text { IX. Análisis de los datos } \\
\text { cuantitativos }\end{array}$ & $\begin{array}{l}\text { VII. Tratamiento e } \\
\text { interpretación de los } \\
\text { resultados }\end{array}$ & $\begin{array}{l}\text { XII. Análisis de resultados. } \\
\text { Discusión }\end{array}$ & $\begin{array}{l}\text { e. Operacionalización } \\
\text { de variables }\end{array}$ \\
\hline
\end{tabular}


(continuación)

\begin{tabular}{|l|l|l|l|}
\hline \multicolumn{1}{|c|}{ Hernández et al. (2010) } & \multicolumn{1}{|c|}{ Alarcón (2008) } & \multicolumn{1}{c|}{ Bernal (2010) } & \multicolumn{1}{c|}{ Valderrama (2014) } \\
\hline $\begin{array}{l}\text { XI. Elaborar el reporte de } \\
\text { resultados }\end{array}$ & $\begin{array}{l}\text { V. Formulación de las } \\
\text { conclusiones }\end{array}$ & & $\begin{array}{l}\text { V. Metodología } \\
\text { de la investigación }\end{array}$ \\
\hline & IX. Redacción del informe & & a. Tipo \\
\hline & & & b. Nivel \\
\hline & & & c. Diseño \\
\hline & & & $\begin{array}{l}\text { d. Método de investigación } \\
\text { e. Población, muestray } \\
\text { muestreo }\end{array}$ \\
\hline & & $\begin{array}{l}\text { f. Técnicas e instrumentos } \\
\text { de recolección de datos }\end{array}$ \\
\hline & & $\begin{array}{l}\text { g. Validezy confiabilidad } \\
\text { de los instrumentos de } \\
\text { recolección de datos }\end{array}$ \\
\hline & & & h. Procesamiento de datos \\
\hline & & & $\begin{array}{l}\text { i. Plan de análisis de } \\
\text { información (datos) }\end{array}$ \\
\hline & & & j. Prueba de hipótesis \\
\hline & & & $\begin{array}{l}\text { Anexo: } \\
\text { Matriz de consistencia }\end{array}$ \\
\hline
\end{tabular}

Elaboración propia

los problemas que ocurren a través de las fuentes de ideas como experiencias, trabajos de investigación, artículos o textos y aportar algún conocimiento que ayude a las entidades involucradas en el problema y a otros investigadores. En Valderrama (2014) se resalta los criterios que deben considerarse en la selección del tema de investigación, entre los cuales están: factibilidad, originalidad, importancia, interés y precisión.

\subsection{Definición del problema de la investigación}

Tanto en Bernal (2010) como en Hernández et al. (2010) se menciona que en la segunda etapa del proceso de investigación se establecen las preguntas, basadas en el análisis del problema. En Valderrama (2014) se subraya que las preguntas de la investigación deben mostrar la relación entre las variables independiente y dependiente, la población en estudio, ubicación y tiempo. En Hernández et al. (2010) se destaca la facilidad que da a la investigación plantearla en la forma de preguntas, las cuales orientan hacia 
las respuestas que se buscan. Las preguntas deben ser precisas y no generales. Estos autores también detallan los requisitos que deben cumplir las preguntas:

- Que no se conozcan las respuestas (si se conocen, no valdría la pena realizar el estudio).

- Que puedan responderse con evidencia empírica (datos observables o medibles).

- Que impliquen el uso de medios éticos.

- Que sean claras.

- Que el conocimiento que se obtenga sea sustancial (que aporte conocimiento a un campo de estudio) (p. 39).

\subsection{Establecimiento de los objetivos generales y específicos}

La tercera etapa se refiere al establecimiento de los objetivos generales y específicos, cuyos logros responden a las preguntas de la investigación. El objetivo general representa la meta o logro general de la investigación, vale decir, está relacionado con el problema general o principal de la investigación. Como se apunta en Valderrama (2014), "los objetivos específicos deben derivarse del objetivo general" (p. 137). Los logros o metas de los objetivos específicos son logros parciales que conducen al cumplimiento del objetivo general. Asimismo, en Valderrama (2014) se muestran algunos ejemplos de verbos de objetivos generales y objetivos específicos. Es importante tener en cuenta lo que sostienen Hernández et al. (2010) sobre los objetivos, que en algunos casos contribuyen a mostrar el proceso para resolver el problema de la investigación indicado y, en otros, comprueba una teoría o genera evidencia empírica que la prueba.

\subsection{Establecer la justificación y delimitación de la investigación}

La justificación significa exponer los motivos de la investigación. Para poder redactarla es necesario considerar tres criterios, según Bernal (2010), que seguidamente se enuncian: el teórico, cuando la investigación genera una reflexión o contrasta resultados sobre el conocimiento existente; el práctico, cuando el desarrollo de la investigación plantea una solución o establece estrategias que contribuyen a resolver el problema de la investigación; y el metodológico, cuando se propone un nuevo método para generar conocimiento válido y confiable.

En relación con el alcance o limitación, Valderrama (2014) señala que es muy importante definir el alcance con claridad y precisión, indicando hasta dónde se profundiza en la investigación. En Bernal (2010) se refieren tres tipos de limitación: la del 
tiempo, en la que se indica el periodo en el que se efectúa el estudio del fenómeno o población objeto de la investigación; la del espacio, que consiste en establecer la frontera geográfica de la investigación; y la de recursos, en la que se puntualizan los recursos financieros para la realización del proyecto de investigación.

\subsection{Definición del tipo de investigación}

En Hernández et al. (2010) se consideran cuatro tipos de investigación: la exploratoria corresponde a investigar problemas que han sido poco estudiados, por lo que este tipo de estudio presenta una perspectiva innovadora; la descriptiva permite identificar las características del objeto de estudio y también muestra las tendencias de una población; la correlacional tiene por objetivo identificar la relación entre dos o más variables, y la explicativa consiste en determinar las causas de los eventos que se investigan.

\subsection{Establecimiento del marco de referencia de la investigación}

En Hernández et al. (2010) se denomina revisión de literatura y desarrollo del marco teórico, es decir, la realización del estado del arte que involucra los métodos secundarios de la investigación, que se refiere a los métodos que permiten recopilar de manera sistemática y rigurosa los estudios primarios relacionados. En una investigación aplicada, tal como se apunta en Genero, Cruz y Piattini (2014), en la ingeniería de software se utilizan revisiones y mapeos sistemáticos de la literatura como métodos de investigación secundarios. De igual modo, cuando no se dispone de estudios primarios que respondan a la pregunta de investigación, se hace necesario realizar un estudio para obtener evidencia empírica.

A su vez, Hernández et al. (2010) señalan que:

Además de un problema bien planteado y sustentado de manera sólida en la teoría y los resultados empíricos previos, se requiere también la utilización adecuada de técnicas de recolección de datos y de análisis estadísticos pertinentes, lo mismo que la correcta interpretación de los resultados con base en los conocimientos que sirvieron de sustento a la investigación. (p. 169)

\subsection{Proceso de realización de la encuesta}

Es una forma de diseño de la investigación que se refiere al "plan o estrategia concebida para obtener la información que se desea" (Hernández et al., 2010, p. 120). Esta etapa se vincula con los métodos primarios, en el que se realizan estudios con 
el propósito de obtener evidencia empírica sobre un tema de interés, según se muestra en Genero et al. (2014).

Según Hernández et al. (2010), el diseño de la investigación puede ser experimental y no experimental. En concordancia con estos autores, este diseño "se realiza sin manipular deliberadamente las variables independientes" (p. 164), y son del tipo transeccional y longitudinal, de acuerdo a la forma de recolectar datos. Genero et al. (2014) sostienen que en las encuestas transeccionales o transversales se obtiene la información de los participantes en un momento determinado y puede ser realizado para estudios exploratorios, descriptivos y correlacionales; mientras las encuestas longitudinales, cuya meta es establecida a largo plazo, teniendo como propósito conocer la evolución a través del tiempo de una determinada población, puede ser realizado para estudios de tendencia, de análisis evolutivo de grupo y de panel, como se expone en Hernández et al. (2010).

De acuerdo con estos autores, a esta etapa se le denomina 'selección de la muestra', y comprende los siguientes pasos:

- definir los casos sobre los cuales se recolectarán los datos

- delimitar la población

- elegir el método de selección de la muestra: probabilístico o no probabilístico

- precisar el tamaño de la muestra requerido

- aplicar el procedimiento de selección, y

- obtener la muestra

\subsubsection{Definir los casos sobre los cuales se recolectarán los datos}

El primer paso en este contexto, definir los casos, consiste en precisar quiénes serán los sujetos objeto de estudio y qué características deberán tener (personas, organizaciones o situaciones y factores), según se indica en Bernal (2010, p. 160).

\subsubsection{Delimitar la población}

En el segundo paso, delimitar la población, es definida como "la totalidad de elementos o individuos que tienen ciertas características similares y sobre las cuales se desea hacer inferencia" (Bernal, 2010, p. 48).

\subsubsection{Elegir el método de selección de la muestra}

El tercer paso es elegir el método de selección de la muestra; según Hernández et al. (2010), para seleccionar las muestras de poblaciones se dispone del muestreo probabilístico y del muestreo no probabilístico. 
El muestreo probabilístico o muestreo aleatorio cuenta con información de las probabilidades de las unidades de análisis seleccionadas en la muestra, lo que permite calcular el grado hasta el cual el valor obtenido de la muestra puede diferenciarse del valor de la población de interés. Se caracteriza por obtener una muestra en forma aleatoria, y esta va a tener la misma probabilidad que cualquier otra muestra (Pérez, 2013). Se identifican, además, varios tipos de muestreo: muestreo aleatorio simple, muestreo sistemático, muestreo estratificado y muestreo por conglomerados.

Por otro lado, el método no probabilístico no permite determinar el error de muestreo; asimismo, no es posible determinar el nivel de confianza de la muestra $y$, finalmente, no permite realizar inferencias sobre la población. En este método se identifican varios tipos de muestreo, entre los cuales se tiene al muestreo por cuotas, muestreo por conveniencia y muestreo de juicio.

Otro tema importante en este contexto es la distribución muestral, que según Pérez (2013, p. 22) es lo que resulta de considerar todas las muestras posibles que pueden tomarse de una población; se clasifica en distribución muestral de medias y distribución muestral de proporciones. En Bernal (2010, p. 179) se indica que el muestreo proporcional se utiliza cuando la variable objeto de la medición se mide en proporciones o probabilidades de ocurrencia.

\subsubsection{Precisar el tamaño de la muestra requerido}

En este paso se procede de la siguiente manera:

- Se identifica si se trata de una población infinita o finita (cuando se conoce el número total de la población N).

- Se determina la proporción de la población que tiene la variable objeto de medición, la cual se puede obtener por medio de resultados de estudios previos o a través de la realización de una muestra piloto. Cuando se estima la proporción de la población mediante la muestra piloto se comienza por construir una tabla de frecuencias, resultado de la medición de la variable objeto del estudio (p).

- Se define el nivel de confianza Z por utilizar, el cual se encuentra en la función de distribución normal tipificada o tabla Z, igual como se procede para el muestreo aleatorio simple. Para efectos de investigación, los valores de Z por utilizar corresponden a niveles de confianza iguales o superiores a $90 \%$.

- Se define el valor del error de estimación E (diferencia máxima entre la proporción muestral y la proporción poblacional que el equipo investigador está dispuesto a aceptar, en función del nivel de confianza definido para el estudio).

- Se calcula el valor del tamaño de muestra según la fórmula correspondiente a la población finita y se concluye sobre el tamaño mínimo de muestra representa- 
tivo, especificando los valores asignados al nivel de confianza (Z), la proporción

(P) y el error de estimación (E) utilizado. Se usa la siguiente fórmula:

$$
n=\frac{Z_{\alpha / 2}^{2} * N^{*} \mathrm{p}^{*} q}{E^{2}(\mathrm{~N}-1)+\mathrm{Z}_{\alpha / 2}^{2} * p^{*} q}
$$

\subsubsection{Aplicar el procedimiento de selección}

Este paso, según Hernández et al. (2010), es importante para la obtención de la muestra; significa aplicar un método para obtener los elementos de la muestra de una manera representativa de la población. Hay tres procedimientos: el primero, denominado la tómbola, radica -según Hernández et al. (2010)- en numerar los elementos y elegir la muestra de una manera aleatoria; el segundo procedimiento es el de números random o aleatorios, consistente en elegir los elementos generando números aleatorios hasta completar la muestra y, por último, el tercer procedimiento, de selección sistemática de elementos muestrales, que se basa en dividir la población en intervalos y elegir los elementos de la muestra aleatoriamente en cada uno de ellos.

\subsubsection{Obtener la muestra}

Esta fase consiste en aplicar el procedimiento de selección de la muestra elegido para extraer la muestra de la población. Puede realizarse mediante un trabajo de escritorio o un trabajo de campo, dependiendo de si se dispone o no de un marco muestral.

\subsection{Recolección de los datos cuantitativos}

Según Bernal (2010), este es un trabajo de campo, a través del cual se prueban las hipótesis, se responde a las preguntas de investigación y se logran los objetivos del estudio originados del problema de investigación.

Según Hernández et al. (2010), la recolección de los datos cuantitativos se efectúa mediante el instrumento de medición, cuyos requisitos son la confiabilidad, validez y objetividad. El proceso para construir el instrumento de medición, de acuerdo a Hernández et al. (2010), sigue once fases, iniciándose con las redefiniciones fundamentales que permiten reevaluar las variables de la investigación, continuada de una segunda fase, que es la revisión enfocada de la literatura, la cual consiste en encontrar instrumentos o sistemas de medición utilizados en otros estudios anteriores.

La tercera fase es la identificación del dominio de las variables por medir y sus indicadores, lo que permite señalar con precisión los componentes de la variable, seguido de la cuarta fase, que se refiere a la toma de decisiones clave si se reúsa un instrumento ya elaborado, o se crea uno nuevo. Si se crea un nuevo instrumento, se presentan tres fases, de la fase 5 a la fase 7, las que no serían necesarias si se opta 
por reusar uno existente, estas fases son: construcción del instrumento, prueba piloto y elaboración de la versión final del instrumento.

En la octava fase se realiza el entrenamiento del personal que va a administrar el instrumento que consiste en entrenar y motivar a los encargados de la aplicación y codificación de las respuestas, seguido de la obtención de autorizaciones para aplicar el instrumento, por parte de personas o representantes de organizaciones que forman parte del estudio; a continuación corresponde la fase de la administración del instrumento, que consiste en la aplicación del instrumento para obtener los datos. La última fase es la preparación de los datos para el análisis, consistente en la codificación, la limpieza y el registro de los datos en una base de datos.

\subsection{Analizar los resultados}

La última etapa consiste en interpretar los hallazgos relacionados con el problema de la investigación, los objetivos propuestos y la hipótesis planteada. Según Bernal (2010), consiste en generar resultados (datos agrupados y ordenados), además de los medios gráficos para la representación de los resultados. En esta etapa se utilizan herramientas computacionales estadísticas para hacer el análisis descriptivo de cada variable del estudio, análisis inferenciales respecto a las hipótesis planteadas y otros, según indican Hernández et al. (2010).

\section{Caso de aplicación del proceso de investigación propuesta}

En esta sección se muestra la aplicación del proceso descrito en la sección 3 de la investigación realizada en el IDIC (Amable et al., 2014), relacionándolo con las etapas mencionadas.

\subsection{Selección del tema de investigación o idea de la investigación}

En la investigación realizada se tuvo en cuenta el tema de caracterización de las mypes productoras de software acerca de la calidad del proceso y del producto software, considerando como antecedente lo señalado en Amable et al. (2014, p. 12), citando a Cruz (2011), donde se realiza el análisis situacional de las mypes a través de un análisis FODA. Entre las fortalezas indicadas, similares en muchas organizaciones, se indica que son incubadoras e instrumentos de aprendizaje y capacitación para el capital humano, característica identificada claramente en las empresas productoras de software, debido al conocimiento y experiencia de los integrantes de este tipo de organizaciones en el desarrollo de software.

Otra fortaleza indicada en Amable et al. (2014, p. 13), haciendo mención a Cruz (2011), es el emprendimiento empresarial que genera creatividad y por tanto innovación, así como factores para la sostenibilidad de la empresa, la cual es también 
una cualidad de las mypes productoras de software, debido a que varios de los emprendedores nacen en las universidades con los conocimientos que adquieren en las aulas e iniciativas que surgen a través de los trabajos que realizan. Sin embargo, hay debilidades en este tipo de organizaciones, que muchas veces tienen dificultades para satisfacer la demanda, tanto en cantidad como en calidad, sobre todo en el aspecto de calidad. Por otro lado, en Amable et al. (2014, p. 13), citando a Pantaleo (2011), se hace referencia al Reporte Standish Group Chaos del año 2004, en el que muestra que los proyectos que terminaron según la planificación original fueron el $31 \%$; los que terminaron fuera de tiempo alcanzaron el $51 \%$, y los proyectos que fueron cancelados alcanzaron el $18 \%$; la mayoría fueron los que terminaron fuera de tiempo. Así también, en Amable et al. (2014, p.13) se cita a Estayno (2009), donde se concluye como resultado del estudio que la mayoría de las empresas tiene menos de diez empleados; su plantel de recursos humanos está formado por profesionales informáticos de alta calificación; las actividades se orientan principalmente al desarrollo de software; las principales dificultades para el crecimiento están relacionadas con el financiamiento y el nivel económico interno; los obstáculos para la exportación están dados por la falta de certificación de calidad, escaso apoyo gubernamental y problemas de comercialización. Se resalta el problema financiero, pero también la necesidad de contar con una certificación de calidad. Es importante precisar que para obtener dicha certificación es necesario que la organización primero adopte mejores prácticas para que cuando acuda la entidad certificadora compruebe que estas organizaciones califican en dicha adopción.

\subsection{Definición del problema de la investigación}

Una de las preguntas de investigación considerada en Amable et al. (2014) fue "¿Cómo la utilización de normas y modelos apoya el proceso de desarrollo de las mypes productoras de software?", que fue la pregunta guía de la investigación realizada.

\subsection{Establecimiento de los objetivos generales y específicos}

En Amable et al. (2014) se indicó el siguiente objetivo específico: "Establecer el nivel de aplicación de normas y modelos de calidad de software" (p. 9); objetivo que permite bosquejar una respuesta al problema de investigación propuesto.

\subsection{Establecer la justificación y delimitación de la investigación}

Con referencia a este aspecto, en Amable et al. (2014) se indica lo siguiente:

Realizar un estudio sobre la situación actual de las empresas mypes productoras de software, sobre todo en el uso de modelos de calidad de software e importancia de la normalización en sus procesos para que sean competi- 
tivas en el mercado tanto nacional como internacional, es importante, para proponer productos de evaluación y gestión que ayuden al desarrollo de dicho sector. (p. 9)

Se informó, de igual manera, que la investigación se llevó a cabo en empresas ubicadas en Lima metropolitana.

\subsection{Definición del tipo de investigación}

De acuerdo con los objetivos propuestos en el estudio de caracterización de las mypes productoras de software (Amable et al., 2014), el tipo de investigación fue el descriptivo.

\subsection{Establecimiento del marco de referencia de la investigación}

En Amable, Millones y Checa (2015) se definieron los conceptos relacionados con los conceptos sobre micro y pequeña empresa, definición de micro y pequeña empresa (mype) y marco legal, problemática de las mypes productoras de software, calidad de software en las organizaciones productoras de software, investigaciones sobre calidad de software, calidad de software en el proceso, metodologías de desarrollo de software, calidad de software en producto de software, pruebas de software, proceso de pruebas, evaluación de la calidad de software, técnicas para obtener información y técnicas de muestreo.

\subsection{Proceso de realización de la encuesta}

En Amable et al. (2014) se aplicó el diseño transeccional para el estudio descriptivo de caracterización de las mypes productoras de software de Lima.

\subsubsection{Definir los casos sobre los cuales se recolectarán los datos}

En la investigación del IDIC (Amable et al., 2014) se consideró entrevistar a los representantes de las mypes productoras de software, principalmente a los gerentes, pues tienen la capacidad de responder a las preguntas que permiten cumplir el objetivo, así como establecer el nivel de aplicación de normas y modelos de calidad. La unidad de análisis es la micro o pequeña empresa productora de software ubicada en Lima metropolitana.

\subsubsection{Delimitar la población}

En Amable et al. (2015) se indica que la población "son todas las empresas micro y pequeñas que son productoras de software ubicadas en Lima 
Metropolitana" (p. 3); con lo cual se delimita adecuadamente a las entidades que formaron parte del estudio.

\subsubsection{Elegir el método de selección de la muestra}

En la investigación de referencia relacionada con las mypes productoras de software (Amable et al., 2014) se consideró un muestreo aleatorio simple como método de selección de la muestra.

\subsubsection{Precisar el tamaño de la muestra requerido}

En Amable et al. (2015) se indicó como marco muestral el listado de micro y pequeñas empresas productoras de software en Lima metropolitana. Para ello se identificaron dos marcos muestrales base, habiéndose obtenido uno de ellos de la Superintendencia Nacional de Aduanas y de Administración Tributaria (Sunat) (272 empresas) correspondiente a la Clasificación Industrial Internacional Uniforme (CIIU,7220-2 (consultores en programas de informática y suministro de programas de informática); y el del sitio web UniversidadPeru.com (57 empresas) correspondiente al CIIU 7210-1 (consultores en equipos de informática), los que hicieron un total de $272+57=329$ empresas de Lima metropolitana.

La distribución se hizo por cantidad de trabajadores, como se muestra en la tabla 2.

\section{Tabla 2}

Distribución por número de trabajadores

\begin{tabular}{cc}
\hline Cantidad de trabajadores & Cantidad de empresas \\
\hline $1-2$ & 125 \\
$3-5$ & 71 \\
$6-10$ & 56 \\
$11-30$ & 54 \\
$31-50$ & 23 \\
\hline Total & 329 \\
\hline
\end{tabular}

Fuente: Amable et al. (2014)

Para el cálculo de la probabilidad de éxito (p), en Amable et al. (2015) se consideró en la prueba piloto analizar el resultado respecto a la pregunta ¿aplican factores de calidad en el desarrollo del producto software?, cuyo resultado fue que un $60 \%$ de los encuestados sí aplicaban factores de calidad, por consiguiente la probabilidad de éxito $(p)$ resultó 0.6, y (q), probabilidad de fracaso, resultó 0.4. 
En la investigación en referencia el nivel de confianza considerado fue del $90 \%$. En una distribución normal este valor corresponde a $1.644\left(Z_{\alpha / 2}=1.644\right)$.

En Amable et al. (2015), para fijar el tamaño de muestra adecuado era preciso determinar el porcentaje de error que se estaba dispuesto a tolerar. Para la investigación, el error de estimación era la diferencia entre la proporción muestral y la proporción poblacional que se estaba dispuesto a aceptar y se consideró un $8 \%$ $(E=0.08)$. En Amable et al. (2015) se eligió a 80 empresas, entre micro y pequeñas empresas productoras de software ubicadas en Lima metropolitana, y para la obtención del tamaño se utilizó la siguiente fórmula.

$$
n=\frac{Z_{\alpha / 2}^{2} * N^{*} \mathrm{p}^{*} q}{E^{2}(\mathrm{~N}-1)+\mathrm{Z}_{\alpha / 2}^{2} p^{*} q}
$$

Aplicando los valores en la fórmula se obtuvo un tamaño de muestra como mínimo de 78, considerando que en las investigaciones a veces no se tiene respuesta de la cantidad prevista, o muchas preguntas están en blanco, lo cual invalida algunas encuestas. Por esa razón, se estimó un 2 \% de no respuesta, obteniéndose como tamaño de la muestra final a 80 empresas, que resultó de multiplicar 78 por 1,02.

\subsubsection{Aplicar el procedimiento de selección}

Aunque la normativa vigente de las mypes indica la identificación de micro y pequeña empresa de acuerdo a sus ingresos, fue difícil obtener dicha información de parte de las empresas y se utilizó una versión anterior, la cual consideraba a la mype relacionada con el número de trabajadores de 1 a 50.

Se realizó la estratificación con el fin de tener representación de las empresas con diferentes tamaños de trabajadores. El procedimiento utilizado en la investigación fue el de selección sistemática de elementos muestrales.

\subsubsection{Obtener la muestra}

Se realizó en forma aleatoria, usando una de las opciones del software Minitab.

\subsection{Recolección de los datos cuantitativos}

Se utilizó el instrumento Cuestionario. Se realizó una primera versión del instrumento de medición Cuestionario, sobre el cual fue probada su pertinencia y eficacia en la fase de prueba piloto.

En esta etapa se pudo obtener una retroalimentación por parte de los encuestados, expertos, para así realizar los cambios necesarios y elaborar la versión final del instrumento.

En Amable et al. (2015, pp. 3-4) se muestran las preguntas del cuestionario utilizado en una sección: 


\section{DATOS DE LA EMPRESA}

Razón social:

RUC:

Dirección:

Clase: ( ) microempresa, ( ) pequeña empresa, ( ) otra clase, ESPECIFICAR:

Persona que proporciona los datos:

Apellidos y nombres:

Puesto:

E-mail de contacto:

Teléfono/celular:

\section{PERSONAL EN LA EMPRESA}

Total de trabajadores:

Número de trabajadores dedicados al desarrollo de software:

¿Requieren de capacitación? Sí ( ) No ( )

Si la respuesta es afirmativa indique el tema de la capacitación requerida:

III. PROYECTOS ANUALES (Marque con una X. Puede marcar más de un tipo)

Desarrollo de software ( ) ¿Cuántos?____ Duración promedio del proyecto:

Servicios ( ) ¿Cuántos?____ Duración promedio del proyecto:

IV. PRODUCTOS (Marque con una X. Puede marcar más de una)

Productos propios ( ) ¿Cuántos?_-___ Especifique los más representativos:

V. CLIENTES (Marque con una X. Puede marcar más de una)

Nacionales ( ) ¿Cuántos?___ Extranjeros ( ) ¿Cuántos?

Si no tienen clientes extranjeros, ¿consideran la oportunidad de exportar sus productos?

Sí ( ) No ( )

VI. CALIDAD DEL PROCESO DE SOFTWARE

Indicar modelos de proceso de software que aplican en el desarrollo de software. Marque con una $\mathrm{X}$.

CMMI ( ) Moprosoft ( ) Competisoft ( )

ISO/IEC 29110 ( ) ISO/IEC 12207（） Otros（） Especifique:

¿Están certificados en algún modelo? Sí ( ) No ( )

Especifique el nombre de la certificación, en caso de respuesta afirmativa:

¿Planean certificarse? Sí ( ) No ( )

VII. CALIDAD DEL PRODUCTO DE SOFTWARE

Indicar estándares de producto de software que aplican. Marque con una X.

Modelo de McCall ( ) ISO/IEC 9126 ( ) ISO/IEC 25000 ( )

Otros ( ) Especifique:

¿Aplican factores de calidad en el desarrollo del producto de software? Sí ( ) No ( )

Si la respuesta es afirmativa, marque con una $X$ los factores de calidad que consideran.

Funcionalidad ( )

Fiabilidad ( )

Eficiencia ( ) Portabilidad ( )

Usabilidad ( )

Facilidad de mantenimiento ( ) Interoperabilidad ( )

Seguridad ( )

Otros ( ) Especifique: 
Adicionalmente, se incluyeron en el cuestionario de la investigación preguntas sobre el proceso de gestión de proyectos, considerando los productos que usualmente se utilizan en dicho proceso y también sobre el desarrollo de software, considerando las prácticas y artefactos utilizados. Las preguntas fueron de tipo cerradas, con múltiples respuestas, como se muestra a continuación.

\section{GESTIÓN DE PROYECTOS}

Señale los productos que utilizan en la etapa de planificación del proyecto:

Enunciado del trabajo ( )

Especificación de desglose de trabajo - EDT ( )

Análisis de documentos ( )

Riesgos del proyecto ( )

Cronogramas ( )

Otros ( ) Especifique:

Señale los productos que utilizan en la etapa de ejecución del plan del proyecto:

Reporte de avance ( )

Solicitud de cambio ( )
Plan de proyecto ( )

Entrevistas ( )

Técnicas de creatividad en grupo ( )

Verificación ( )

Presupuestos ( )
Acta de reunión ( )

Otros ( ) especifique:

Señale los productos que utilizan en la etapa de evaluación y control del proyecto:

Acciones correctivas ( )

Software de monitoreo ( )

Otros ( ) Especifique:

Señale los productos que utilizan en la etapa de cierre del proyecto:

Configuración del software ( ) Repositorio del proyecto ( )

Acta de aceptación ( )

Otros () Especifique:

IX. PROCESO DE DESARROLLO DE SOFTWARE. (Puede marcar más de una vez)

Señale las metodologías o prácticas que utilizan en el proceso de desarrollo de software:

$\operatorname{RUP}($ ) Scrum () Kanban ()

$\operatorname{TDD}() \quad$ Lean ( ) Scrum/XP ( )

Scrumban ( ) Programación extrema ( ) Otros ( ) Especifique:

Señale los artefactos que utilizan durante el desarrollo de sus productos de software:

Diagrama de procesos ( )

Diagrama de casos de uso ( )

Diagrama de actividades ( )

Diagrama entidad - relación ( )

Diagrama de clases ( )

Diagrama de navegación ( )

Diagrama de colaboración / comunicación ( )

Diagrama de secuencia ( )

Diagrama de despliegue ( )

Historias de usuario ( )

Product backlog ( )

Reunión de planificación del sprint ( )

Sprint backlog ( )

Reunión de revisión del sprint ( )

Retrospectiva del sprint ( )

Daily meeting ( )

Otros ( ) Especifique: 
La siguiente fase consiste en realizar el entrenamiento, en la investigación se elaboró el "Manual de instrucciones del encuestado y/o registrador", que sirvió de base para el entrenamiento y uso en la realización de la encuesta.

La fase de la investigación "Obtener autorizaciones para aplicar el instrumento" consistió en contactar telefónicamente con el gerente o representante de la empresa para informarle sobre la importancia de su participación y colaboración en este estudio. Esta labor resultó ardua, debido a que el trabajo relacionado a la producción de software fue muy exigente. Sin embargo, se pudo conseguir que atendieran la realización de la encuesta por un lapso de media hora. La siguiente fase es la administración del instrumento, que consistió en la aplicación del cuestionario de la encuesta en la fecha y hora acordada.

Se comprometió a que los encuestadores acudieran a las citas pactadas en la fecha y hora acordadas. La siguiente fase fue la administración del instrumento, que consistió en la aplicación del cuestionario de la encuesta.

Para la preparación de los datos, que incluye la codificación, limpieza e ingreso en la base de datos, se usó el software SPSS.

\subsection{Analizar los resultados}

En Amable et al. (2015) se presentó el resultado de las clases de empresa, a través de una gráfica pastel (pie), el cual se puede apreciar en la figura 1:

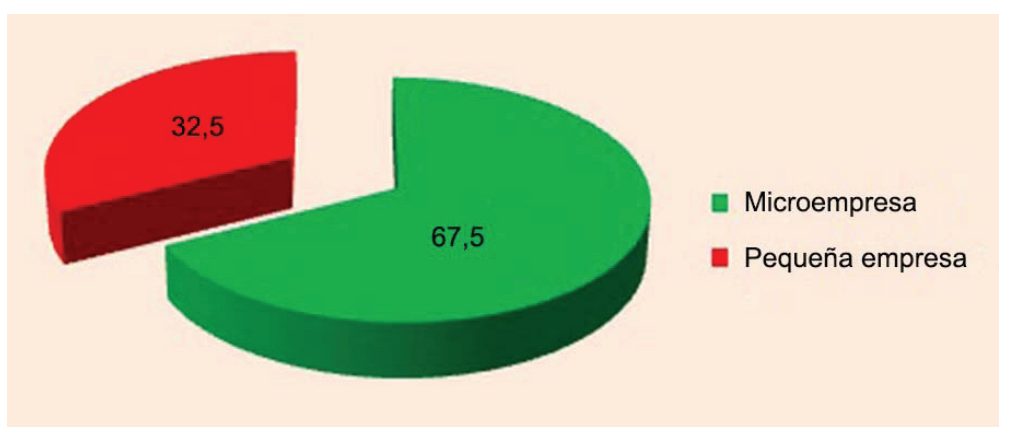

Figura 1. Clase de empresa

Fuente: Amable et al. (2015)

Asimismo, en Amable et al. (2015) se presentó, a través de un histograma, el resultado relacionado con el uso de los modelos de procesos de software que aplican en el desarrollo de software (véase la figura 2). 


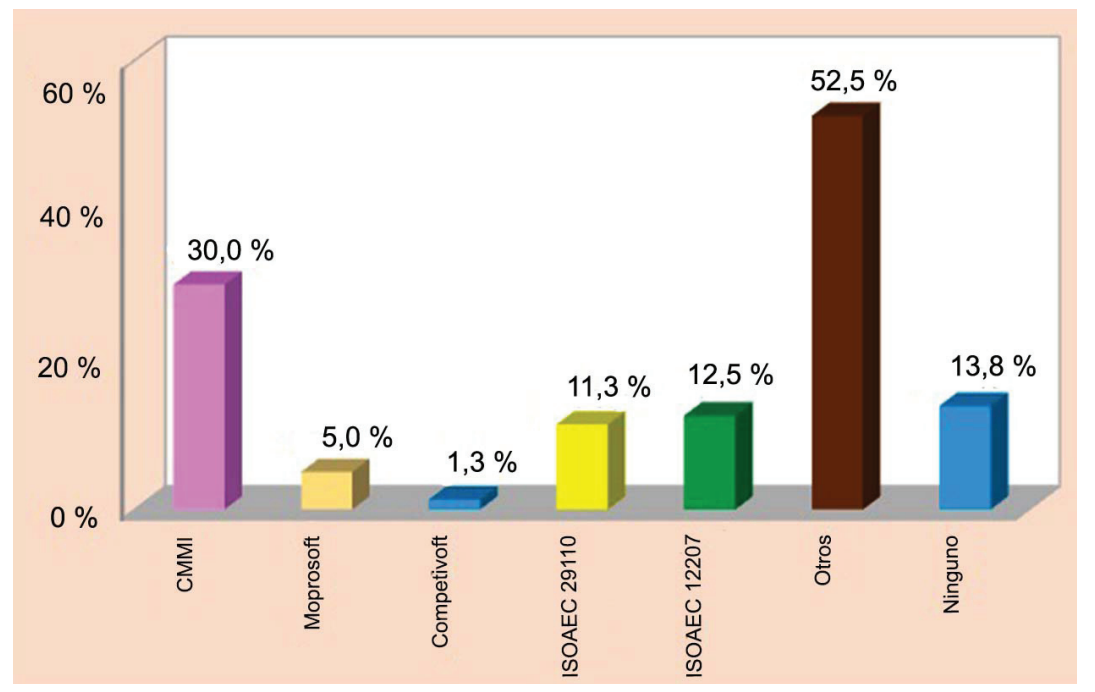

Figura 2. Modelos de procesos de software

Fuente: Amable et al. (2015)

Según se muestra en la figura 3, se crearon histogramas para el proceso de las preguntas sobre gestión de proyectos, respecto a los productos utilizados en el subproceso de planificación del proyecto.

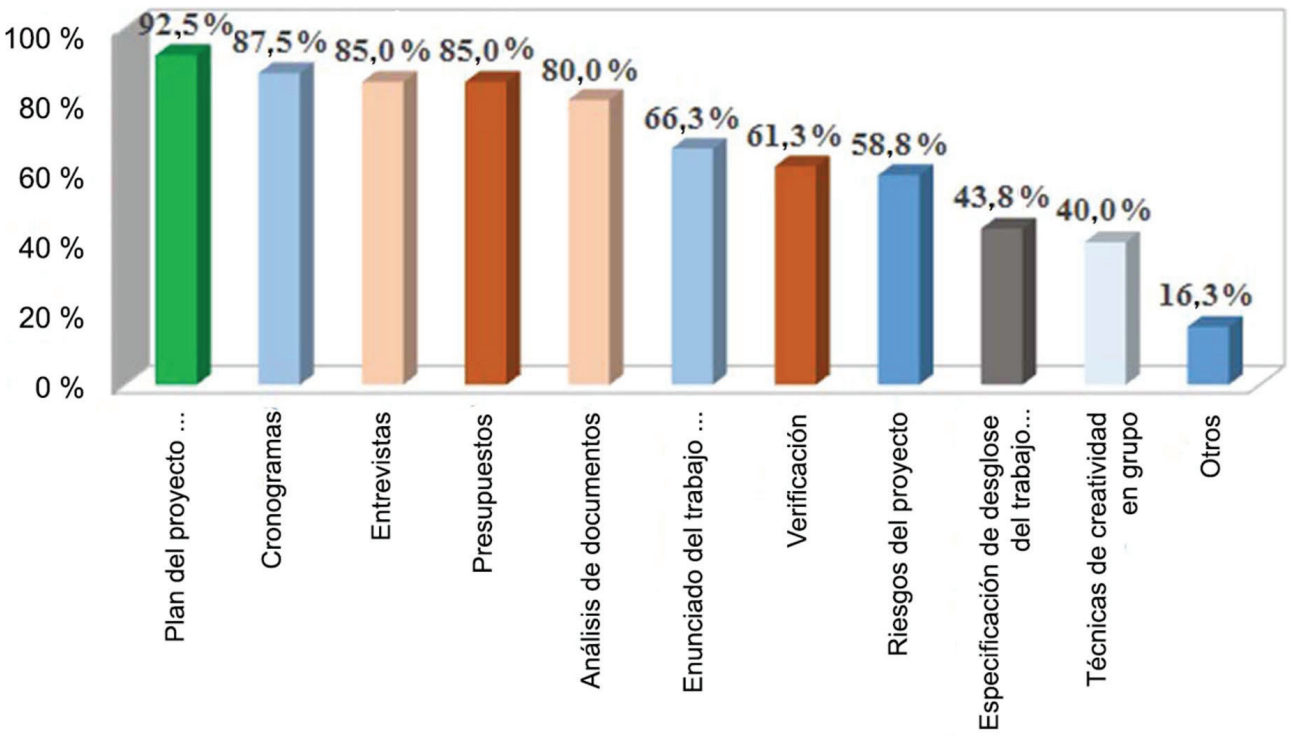

Figura 3. Productos de la planificación del proyecto

Fuente: Amable et al. (2014) 
Asimismo, en relación al proceso del desarrollo de software se obtuvo el histograma de las metodologías o prácticas utilizadas por las empresas encuestadas, según se observa en la figura 4.

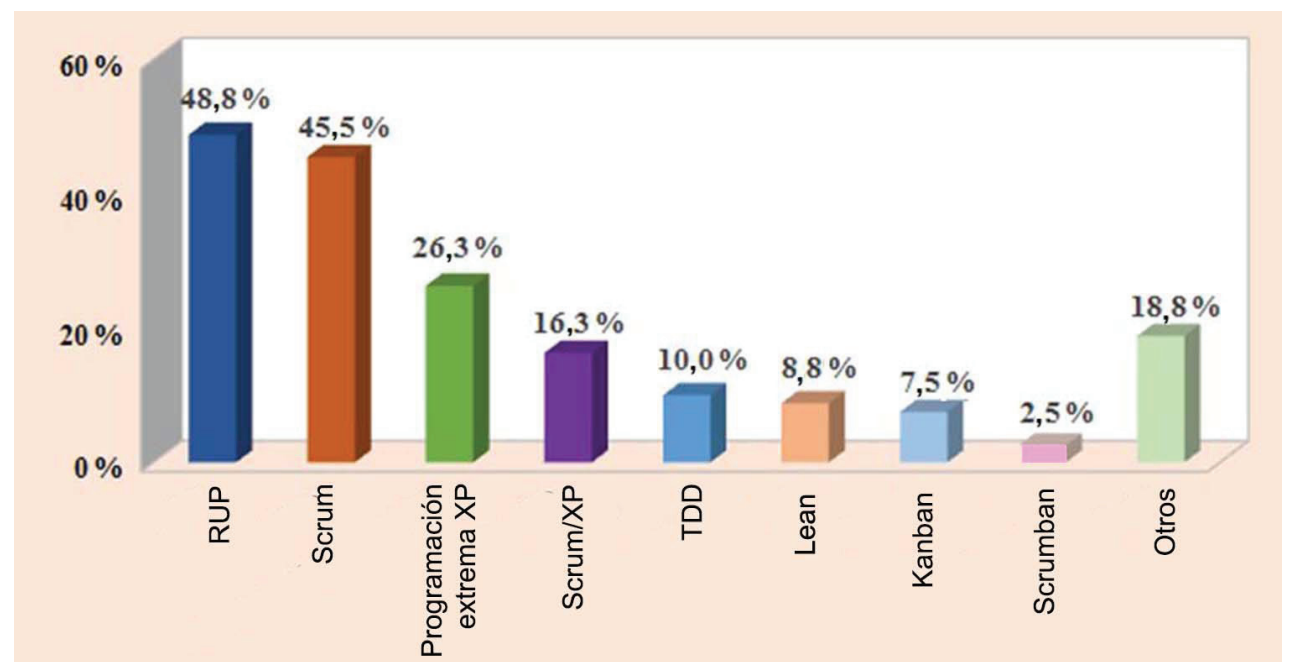

Figura 4. Metodologías/Prácticas de desarrollo del software Fuente: Amable et al. (2014)

En relación a la interpretación de los hallazgos vinculados con el problema de la investigación, los objetivos propuestos y la hipótesis planteada en la investigación, según se indica en Amable et al. (2015), se señaló lo siguiente:

a) La población es mayormente representada por microempresas, con el 67,5\%.

b) El 52,5 \% de las mypes encuestadas manifestaron utilizar otros modelos de calidad de proceso del desarrollo de software, los cuales eran metodologías o formas propias de hacer software; además, había un 13,8 \% que no utilizaba ningún modelo de calidad de proceso. Sumados ambos porcentajes, un 66,3\% de las empresas encuestadas no consideraba la utilización de los modelos de calidad de proceso más conocidos en el mercado. Esto se demostraba en el resultado, en el cual se observaba el bajo uso y conocimiento de los estándares de calidad en las mypes.

c) El 27,6\% de las empresas aplicaba estándares de calidad de producto software; sin embargo, había un 42,5 \% que empleaba sus propios métodos y un 38,8 \% que no utilizaba ningún modelo. Se concluyó que muy pocas empresas conocían los estándares de calidad de producto y por tal razón no los aplicaban.

Como consecuencia del análisis de los resultados de las preguntas sobre los procesos de gestión de proyectos y desarrollo de software, Amable et al. (2014) determinaron lo siguiente: 
a) En relación con el subproceso planificación del proyecto se reparó en que los productos más usados en esta etapa eran plan del proyecto, el 92,5\% de las empresas; cronogramas, el 87,5; entrevistas y presupuestos, un 85 \% y análisis de documentos, un $80 \%$.

b) Respecto al proceso de desarrollo de software, en las metodologías seguidas se observó que el 48,5 \% utilizaba RUP, el 45 \% SCRUM, y el 26,3 \% Programación Extrema XP. Son menos las empresas que empleaban SCRUM/XP, TDD, Lean, Kanban y Scrumban. El 18,8 \% hacía uso de otras metodologías.

c) En relación con las metodologías y prácticas de desarrollo de software, casi el $50 \%$ de los encuestados manifestó utilizar RUP, que es una metodología de tipo prescriptiva. Sin embargo, se mostró una tendencia a usar las metodologías ágiles, que son de tipo adaptativo, principalmente SCRUM.

4.

\section{Conclusiones}

a) Para obtener un resultado confiable y de calidad, es necesario seguir el proceso formal de investigación cuantitativa. Existen varios textos que relatan el proceso, pero es indispensable elegir uno que sirva de guía en todo el proceso de la investigación.

b) Una vez elegido el proceso hay que seguirlo con rigurosidad, es decir, no dejar de hacer ninguna de las etapas indicadas. Esto garantiza resultados confiables.

c) En lo referente a la etapa de la realización del proceso de la encuesta, puede ocurrir que no se disponga de la muestra exigida según el cálculo realizado, o que existan diversas dificultades. Es necesario solucionar dichas dificultades, ya que no se puede reducir la cantidad de entrevistados.

d) Cuando uno de los objetivos específicos es determinar la caracterización de la población, se debe seguir el proceso de investigación cuantitativa, tal como se realizó en la investigación ejemplificada en el artículo.

\section{Referencias}

Alarcón, R. (2008). Métodos y diseños de investigación del comportamiento. Lima: Universidad Ricardo Palma.

Amable, C. M., Millones, R. R., y Checa. F. R. (2014). Análisis del uso de modelos de calidad de software. Una propuesta de mejora de procesos en las mypes productoras de software de Lima. Lima: Universidad de Lima, Instituto de Investigación Científica (IDIC).

Amable, C. M., Millones, R. R., y Checa, F. R. (2015). Calidad de software en las mypes productoras de software en Lima. (Ponencia). VII Congreso Internacional de 
Computación y Telecomunicaciones. Memoria COMTEL 2015 (pp. 133-139). Lima: Universidad Inca Garcilaso de la Vega, Fondo Editorial.

Bernal, T. C. A. (2010). Metodología de la investigación. México: Pearson.

Genero, M., Cruz, J. A., y Piattini, M. G. (2014). Métodos de investigación en ingeniería del software. Madrid: Ra-Ma.

Hernández, R., Fernández, C., y Baptista, P. (2010). Metodología de la investigación. México, D. F.: McGraw-Hill.

Pérez, R. (2013). Estadística aplicada: para ciencias económicas, administrativas y sociales. México, D. F.: Trillas.

Valderrama, S. (2014). Pasos para elaborar proyectos de investigación científica: cuantitativa, cualitativa y mixta. Lima: San Marcos. 